\title{
Fractal Art: \\ Closer to Heaven? \\ Modern Mathematics, the art of Nature, and the nature of Art
}

\author{
Charalampos Saitis \\ Music \& Media Technologies \\ Dept. of Electronic \& Electrical Engineering \\ University of Dublin, Trinity College \\ Printing House, Trinity College \\ Dublin 2, Ireland \\ saitisc@tcd.ie
}

\begin{abstract}
Understanding nature has always been a reference point for both art and science. Aesthetics have put nature at the forefront of artistic achievement. Artworks are expected to represent nature, to work like it. Science has likewise been trying to explain the very laws that determine nature. Technology has provided both sides with the appropriate tools towards their common goal. Fractal art stands right at the heart of the art-science-technology triangle. This paper examines the new perspectives brought to art by fractal geometry and chaos theory and how the study of the fractal character of nature offers promising possibilities towards art's mission.
\end{abstract}

\section{Introduction}

"When I judge art, I take my painting and put it next to a God-made object like a tree or flower. If it clashes, it is not art."

Paul Cézanne [1]

Mathematics has always been affecting art. Mathematical concepts such as the golden ratio, the platonic solids and the projective geometry have been widely used by painters and sculptors whereas the Pythagorean arithmetic perception of harmony dominates western music to this very day. Infinity and probability theory inspired artists such as M. C. Escher and composers such as Iannis Xenakis forming trends in contemporary art and music. The evolution of technology created new areas of intersection between mathematics and art or music: digital art, computer music and new media.

The founding of chaos theory introduced mathematics to a fascinating and intriguing new reality: nature itself. Until then, scientists were only capable of observing the nonlinear dynamical character of the natural structures and processes. Now they had mathematical tools, which described, explained and proved the chaotic properties of nature, like the weather systems and the butterfly effect. Computers became an integral part of this scientific revolution, being capable of calculating nonlinear dynamical systems and visualizing the results over time; scientists were able to watch the chaotic evolution of a naturally-occurring phenomenon on a computer's screen. Eccentric shapes, irregular geometrical objects and extraordinary figures emerged, revealing a world where the unreal (graphic representations of algorithmic processes) implied the real (nature). Benoit Mandelbrot was the first mathematician to shape this new area into an individual self-standing theory, which instantly became the most popular of all. He introduced the neologism fractal to unite all these strange objects under one term. "I coined fractal from the Latin adjective fractus. The corresponding Latin verb frangere means 'to break'; to create irregular 
fragments. It is therefore sensible -and how appropriate for our needs!- that, in addition to 'fragmented' (as in fraction or refraction), fractus should also mean 'irregular', both meanings being preserved in fragment." [2]

Artists were instantly attracted to fractals. Artistic interest burst out, resulting in a new form of digital art, which rapidly became popular both inside and outside the artistic and scientific communities. Musical interest followed almost simultaneously, focused mainly on the area of algorithmic composition. Consequently challenging questions arose: Is fractal art the next big thing? Is fractal art the fulfilment of art's mission? Are we getting closer to Heaven?

The following sections of the paper will discuss some initial concepts in a mathematical and philosophical approach. Section 1 will introduce the general mathematical notions of chaos theory and fractal geometry. Section 2 will introduce fractal art, its areas and sub-areas and their mathematical origins. Section 3 will outline a brief background on the pre-existing use of fractal structures in artworks. Section 4 will discuss the view that the geometry of nature is neither Euclidean nor projective but mainly fractal and chaotic, and how this implies that fractal art is (promised to be) the art of nature.

\section{What Is A Fractal Anyway?}

Fractal Geometry (and some Topology). The topological dimension of a set is defined as the number of independent parameters needed to describe a point in the set. For example, a point in the plane is described by two independent parameters (also known as the Cartesian coordinates of the point), so in this sense, the plane is two-dimensional. By definition topological dimension is always a natural number. However, topological dimension behaves in quite unexpected ways on certain highly irregular sets such as fractals. Box dimension gives another way to define dimension for such sets.

Let $F$ be a fractal in the three-dimensional space and $\mathrm{N}(r)$ the minimum number of boxes (i.e. cubes) of radius less than or equal to $r$ required to completely cover $F$ in terms of its volume. Clearly, as $r$ gets smaller $\mathrm{N}(r)$ gets larger. Very roughly, $\mathrm{N}(r)$ is found to be proportional to $1 / r^{d}$ as $r \rightarrow 0$, where $d$ is a real number. Then $d$ is the Box dimension of $F$. Box dimension measures the space-filling ability, i.e. it refines the concept of topological dimension by relating it to other properties of the space such as volume.

Though unexpected, Box dimension is a real number, more often a fraction. Fractals and highly irregular sets do not have an integer dimension but a fractional dimension. There are various closely related notions of possibly fractional dimensions. They are usually referred to as fractal dimensions. Note that 'fractal' is a neologism and as such is not semantically related to 'fractional'. Mandelbrot formally defined a fractal to be a set with Box dimension strictly greater than its topological dimension. However it proved to be unsatisfactory in that it excluded a number of sets that clearly ought to be regarded as fractals. As Kenneth Falconer notes, "the definition of a 'fractal' should be regarded in the same way as the biologist regards the definition of 'life' [...] just a list of properties characteristic of a living thing [...] most living things have most of the characteristics on the list, though there are living objects that are exceptions to each of them. In the same way, it seems best to regard a fractal as a set that has (a list of) properties, rather than to look for a precise definition, which will almost certainly exclude some interesting cases." [3] Therefore, a fractal $F$ is (defined as) a geometrical object that generally has the following features:

1. $F$ has a fine structure, i.e. detail on arbitrarily small scales.

2. $F$ is too irregular to be described in traditional geometrical language, both locally and globally.

3. $F$ has some form of self-similarity, at least approximately or stochastically.

4. $F$ has a 'fractal dimension' (defined in some way) that is greater than its topological dimension. 
5. $F$ has a simple and recursive definition in most cases of interest.

Fractals are generally generated following three techniques:

1. Escape-time fractals: defined by a recurrence relation at each point in a space.

2. Iterated function systems (IFS): a fixed geometric replacement rule exists.

3. Random fractals: these are generated by stochastic rather than deterministic processes.

Fractals can also be classified according to their self-similarity. Three types of self-similarity are identified in fractals, listed below in a direct correspondence to the generation techniques presented above:

1. Quasi-self-similarity: the fractal appears approximately (but not exactly) identical at different scales. Quasi-self-similar fractals contain small copies of the entire fractal in distorted and degenerate forms. This is a loose form of self-similarity.

2. Exact self-similarity: the fractal appears identical at different scales. This is the strongest type of selfsimilarity.

3. Statistical self-similarity: the fractal has numerical or statistical measures which are preserved across scales. This is the weakest type of self-similarity.

Chaos Theory. Chaos theory describes the behaviour of systems of nonlinear dynamical equations when iterated. Iteration refers to the process, whereby an initial value is input to a system of equations and the output is fed back into the system as a new input value. The same process is repeated in infinitely many steps. Each step provides a value, which represents a point in $n$-dimensional space ( $n$ being determined by the number of variables in the equations). The orbit of the system is defined as the set of these points over time. An attractor of the system is a set to which the orbit of the system converges. There are three categories of behaviour into which the system can fall upon iteration: constant, where all points in the orbit tend towards a stable value; oscillatory, where all points in the orbit belong to a repeating set; and chaotic, where no point in the orbit is visited twice in a finite time period. The last category of behaviour is the most interesting. The system wanders around a range of points, often returning to close, but never identical points and its attractor, being seen from a geometrical point of view, is a complicated set with fractal characteristics called a strange attractor or a fractal attractor. Strange attractors are actually fractals, belonging to the most interesting and beautiful ones. The most essential feature of a strange attractor is its sensitivity to the initial conditions, i.e. minor differences in the initial input values of the system can produce drastic, unexpected results after a certain number of iterations. [4] In other words: chaos!

Historically, mathematical 'strange' structures existed before fractals. They were characterized as 'pathological' since they did not fit the patterns of Euclid. However, "the mathematicians who created (them) regarded them as important in showing that the world of pure mathematics contains a richness of possibilities going far beyond the simple structures that they saw in Nature. [...] Now, as Mandelbrot points out, $[\ldots]$ Nature has played a joke on mathematicians. The $19^{\text {th }}$-century mathematicians may have been lacking in imagination, but Nature was not. The same pathological structures that the mathematicians invented to break loose from $19^{\text {th }}$-century naturalism turn out to be inherent in familiar objects all around us." [5]

Scaling self-similarity is the most critical property of a fractal; it is the 'strange attractor' of the dynamical system defined by mathematics, art and nature. 


\section{Welcome to Fractaland!}

"Just as the creation of a fractal structure involves the process of iteration, so the production of artistic works involves iteration. The creative process is a system wherein the output eventually becomes part of the input. In this way, the process of making art becomes self-similar, self-referential and an iteration of itself."

Edward Berko [6]

Fractal art is created by calculating fractal mathematical functions (algorithms) and convert the results into digital still or animated images and music. Fractal images are graphs of the results while fractal music maps the calculation results to music pitches or other sounds. Although the initiative of the artist/programmer is what defines the resulted artwork, Fractal art has been strongly criticized as to whether or not it should be considered a form of art because of its computational origins.

Fractal images are digital images that either consist of a single fractal or are composed of several fractal and non-fractal objects. Software tools have become widely available, whereby the artist is able to algorithmically generate a fractal, to apply colour patterns and to compose digital images. The algorithms used fall under the three generating techniques presented in the previous section, thus allowing the artist to decide on the type of self-similarity and the level of complexity. One of the most popular fractal generating programmes is Ultra Fractal. Its innovating feature is the built-in layering capability; in addition it was the first programme to place total control of the image in the hands of the user. It should be noted here that colouring a fractal image is an essential part of the process. Geometrically, a fractal object consists of 'neighbourhoods' of different importance. Computers generate a fractal by applying different colours to different levels of importance, the result being outstanding colourful images that challenge imagination.

Two sub-areas of fractal images are fractal flames and fractal landscapes. Fractal flames are mainly generated with IFS, whereas fractal landscapes by stochastic algorithms. Furthermore, sequencing fractal images creates fractal animations.

Fractal music is an area of algorithmic composition. Fractal generating algorithms are applied to pitch, dynamics, duration, time and other audio parameters to determine compositional processes. There are several types of fractal music according to the methods and software used. Several existing programmes generate music using solely fractal mathematics, and transformations to fit a chosen musical scale. However, the majority of the software uses fractal as well as a great many other algorithmic techniques combined. In other cases, composers use fractal compositional processes such as structural self-similarity as a starting point for further development using conventional instruments, samples and other sounds.

Another way of composing fractal music is using iterated systems of nonlinear dynamical equations as note generating algorithms. The result is directly related to the behaviour of the system. Obviously, constant behaviour will produce no interesting musical results. Oscillatory behaviour has the possibility of producing interesting repetitions, if the period (the number of distinct points in the repeating set) is large enough, but in practice the set is usually rather small, resulting in melodies that circle between only a few pitches. It is the chaotic behaviour that holds the most musical interest. When the strange attractor is interpreted musically, the result is very similar to variations on a theme. The material produced has a high degree of correlation with its past, but is always producing something new at the same time. 


\section{Fractals Have Always Been There!}

"Since a long time I am interested in patterns with 'motives' getting smaller and smaller till they reach the limit of infinite smallness."

M. C. Escher [7]

Well before the mathematical establishment of fractals and chaos, fractal patterns, self-similarity, chaotic structures and infinity have been used by painters, sculptors and composers as both motivation and quantitative tools.

Jackson Pollock created some abstract paintings in the late 1940s, in which he used two revolutionary methods both implying chaotic patterns. In the first he used his whole body to introduce a wide range of length scales into his painting motion. In the second he applied paint by letting it drip on the canvas. Furthermore, electronic de-construction of Pollock's paintings into their constituent coloured layers showed that each of the individual layers consist of a uniform, fractal pattern. [8] Escher created his famous tessellation drawings inspired by the notions of infinite repetition and scaling self-similarity, often producing optic illusions. Salvador Dali's La Visage de la Guerre depicts a fractal progression of ever smaller death masks.

Self-similarity abounds in canonical works of western music, present in different forms at all time periods. Canons and fugues are the main examples. A canon is a contrapuntal composition that employs a melody with one ore more imitations of itself played after a given duration (usually one measure). The initial melody is called the leader, while the imitative the follower. The follower is either an exact replica of the leader or a transformation, thus there are several different types of canons. A fugue is a more complex form of contrapuntal composition where one main theme, the subject, sounds in successive imitation in each voice. Studies on J. S. Bach's famous canons and fugues prove the masterly use of selfsimilarity and scaling. The music of Beethoven and Mozart also consists of similar elements. As Mandelbrot pointed out, "music displays fractal characteristics because of its inherently hierarchical nature.” [9] Does 'nice', 'harmonious' music require long-range dependence or self-similarity?

\section{It Is A Fractal's World After All, Isn't It?}

"The observation by Mandelbrot of the existence of a 'Geometry of Nature' has led us to think in a new scientific way about the edges of clouds, the profiles of the tops of forests on the horizons, and the intricate moving arrangement on the wings of a bird as it flies."

Michael F. Barnsley [10]

With the establishment of fractal geometry and chaos theory it soon became clear that these are useful mathematical tools for describing nature. Scientists went on examining natural patterns and objects from a completely new angle, the results being more than just interesting. Irregularity, chaos, abrupt changes, discontinuity, self-similarity, scaling: all rule both the inner and outer beauty and harmony of nature and life. Trees, branches, leaves, the roots of a plant, cauliflowers, snowflakes, diamonds, coastlines, mountains, clouds, stars, the sky, galaxy clusters: fractal attractors describe visible natural shapes. The weather, the solar system, plate tectonics, turbulent fluids, population growth, economy: examples of chaotic dynamical systems. The brain and bronchial lobes are also examples of bodily structures with elements of self-similarity and scaling.

In 1982 Benoit Mandelbrot wrote his fundamental essay The Fractal Geometry of Nature, in which he introduced his revolutionary ideas in a mathematical, philosophical and artistic way, being considered 
as a bible for both scientists and artists. The innovative concept of Mandelbrot was to usefully represent a natural pattern by a fractal set. As Mandelbrot says, "many facets of Nature can only be described with the help of fractals [...] Nature's patterns are irregular and fragmented [...] self-similarity is [...] the fabric of Nature." [11]

Chaos theory brought out a whole new aesthetic in science. Traditionally, science has considered the relation between the observer and the observed to be purely 'objective'. However, chaos dramatically negated this assumption by showing that this relation is chaotic dynamical and thus 'subjective'. Artists on the other hand have always understood that a change in one small part of a painting or a musical composition may destroy or transform the work. Chaos united the two worlds of art and science towards a further understanding of nature. [12]

Furthermore, the American Heritage Directory defines art as "1. Human effort to imitate, supplement, alter or counteract the work of nature and 2. The conscious production or arrangement of sounds, colours, forms, movements, or other elements in a manner that affects the sense of beauty, specifically the production of the beautiful in a graphic or plastic medium." [13] Fractal art obviously follows this definition in a more persuasive way than other forms of art, since it originates from nature. Nature forms patterns somewhere between order and chaos, some of them being orderly in time but disorderly in space, others orderly in space but disorderly in time: fractal patterns. [14] We meet these patterns everywhere in the real world we live in. Even the way humans react to natural phenomena is in most cases unpredictable. Art has been either totally orderly (classicism, cubism) or totally disorderly (dada, surrealism); with fractal art the in between gap is filled in.

In the field of music, fractal algorithms are expected to produce more realistic natural sounds and subtle melodies than conventional algorithmic approaches. Music produced by chaotic nonlinear dynamical systems is highly acclaimed as more aesthetically interesting than music obtained with other stochastic algorithms such as Markov Chains. [15] Fractal music yields patterns that originate from natural procedures, thus being harmonious and beautifully complex. Further processing of these patterns, either as succession of musical events or as complex frequency spectra, promises to produce musical material, which will bring new horizons in composition. As fractal structures are identified in nature, in the nature of sound itself, and in our perception of musical beauty, fractal music is about to be the next musical revolution. [16]

\section{Conclusion}

Mathematics is on the artistic side a creation of new rhythms, orders, designs, harmonies, and on the knowledge side, is a systematic study of various rhythms, orders, designs and harmonies.

William L. Schaaff [17]

Continuity and infinity characterize nature itself. Therefore image and sound, as integral parts of nature, and consequently art and music, could be redefined through them, taking on a completely new vision and mission. Modern mathematics provides the necessary tools, both scientific and artistic, through fractal geometry and chaos theory. Fractal art is located at the very point where the nature of Art meets the art of Nature; its infinite (but not self-similar!) possibilities are yet to be explored. 


\section{References}

[1] http://www.myspace.com/paul_cezanne

[2] Benoit B. Mandelbrot, The Fractal Geometry of Nature, New York: W. H. Freeman \& Co, 1982, pp. 4

[3] Kenneth Falconer, Fractal Geometry, Mathematical Foundations and Application, Chichester: John Wiley \& Sons, revised edition, 2002, pp. xx-xxi

[4] Gavin O'Brien, Master Thesis, A Study of Algorithmic Composition and its potential for aiding laptop-based interactive performance, M.Phil. in Music \& Media Technologies, University of Dublin, Trinity College, 2004, pp. 22-23

[5] Mandelbrot, pp. 3-4

[6] John Briggs, Fractals, The patterns of Chaos. Discovering a New Aesthetic of Art, Science and Nature, London: Thames and Hudson, 1992, pp. 168

[7] Briggs, pp. 166

[8] Richard Taylor; Adam Micolich; David Jonas, Fractal Expressionism. Physics World, 12/10, 1999

[9] O’Brien, pp. 26

[10] Michael F. Barnsley, Fractals Everywhere, Boston; London: Academic Press Professional, 1993, pp.1

[11] Mandelbrot, pp. 193-194, 1, 423

[12] Briggs, pp. 31-33

[13] Jane Parke, Fractal Art - A Deliberate Approach, 2000, http://www.infinite-art.com/ylem.html

[14] Kristine H. Burns, Doctoral Dissertation, The History and Development of Algorithms in Music Composition 1957-1993, Doctor of Arts, Ball State University, 1994, pp. 7

[15] O’Brien, pp. 26-27

[16] Julie A. Scrivener, "Proceedings of Bridges 2000: Mathematical Connections in Art, Music, and Science" [July 28-30, 2000], Applications of Fractal Geometry to the Player Piano Music of Conlon Nancarrow, ed. Reza Sarhangi, Winfield, Kan. Southwestern College, 2000, pp. 185-192

[17] Robert E. Kennedy; Curtis N. Cooper, Bach, 5465 and Upside-Down Numbers, The College Mathematics Journal, Vol. 18, No. 2. (Mar., 1987), pp. 111-115. 